\title{
Physical Activity and the Risk of Liver Cancer: A Systematic Review and Meta-Analysis of Prospective Studies and a Bias Analysis
}

\author{
Sebastian E. Baumeister, Michael F. Leitzmann, Jakob Linseisen, Sabrina Schlesinger
}

See the Notes section for the full list of authors' affiliations.

Correspondenceto: Sebastian E. Baumeister, PhD, Epidemiology, Ludwig-Maximilians-Universität München, UNIKA-T Augsburg, Neusässer Str. 47, 86156 Augsburg, Germany (s.baumeister@unika-t.de).

\begin{abstract}
Background: Physical inactivity is an established risk factor for several cancers of the digestive system and female reproductive organs, but the evidence for liver cancers is less conclusive.

Methods: The aim of this study was to synthesize prospective observational studies on the association of physical activity and liver cancer risk by means of a systematic review and meta-analysis. We searched Medline, Embase, and Scopus from inception to January 2019 for prospective studies investigating the association of physical activity and liver cancer risk. We calculated mean hazard ratios (HRs) and 95\% confidence intervals (CIs) using a random-effects model. We quantified the extent to which an unmeasured confounder or an unaccounted selection variable could shift the mean hazard ratio to the null.

Results: Fourteen prospective studies, including 6,440 liver cancers, were included in the systematic review and meta-analysis. The mean hazard ratio for high compared with low physical activity was 0.75 ( $95 \% \mathrm{CI}=0.63$ to $0.89 ; 95 \%$ prediction interval $=0.52$ to $\left.1.07 ; I^{2}=64.2 \%\right)$. We estimated that $67.6 \%(95 \% \mathrm{CI}=56.6 \%$ to $78.5 \%)$ of all true effect estimates would have a hazard ratio less than 0.8. Bias analysis suggested than an unobserved confounder would have to be associated with a 1.99-fold increase in the risk of physical activity or liver cancer to explain away the observed mean hazard ratio. An unaccounted for selection variable would have to be related to exposure and endpoint with a relative risk of 1.58 to explain away the mean hazard ratio.

Conclusions: Physical activity is inversely related to the risk of liver cancer. Further studies with objectively measured physical activity and quasi-experimental designs addressing confounding are needed.
\end{abstract}

Worldwide, liver cancer was the fourth leading cause of cancer death in 2015 after lung, colorectal, and stomach cancer (1). The most common type of primary liver cancer is hepatocellular carcinoma. The burden of liver cancer varies markedly by sex and geographic region (2). Major risk factors include infections (hepatitis $B$ virus, hepatitis $C$ virus), aflatoxins, metabolic factors (diabetes, excess body fatness), and behavioral factors (alcohol consumption, tobacco) $(3,4)$. In the past several decades, we have witnessed an increase in the incidence of liver cancers in Western countries $(1,5)$. Obesity and physical inactivity have emerged as risk factors for a number of cancers of the digestive system and female reproductive organs $(6,7)$. Based on current knowledge, factors related to energy balance, encompassed by physical activity and obesogenic effects of diet, are estimated to account for $10 \%-15 \%$ of the overall cancer burden (8). A previous systematic review and meta-analysis found an inverse association between total physical activity and liver cancer risk (9). However, that systematic review and meta-analysis (9) was not comprehensive, because it did not include a recent multinational cohort study (10).

Nonetheless, as stated by the World Cancer Research Fund International (7) and an umbrella review (6), the existing evidence is inconclusive to support a beneficial effect of physical activity for lowering the risk of liver cancer. Moreover, metaanalysis of observational studies might exaggerate bias because of confounding by increasing statistical power and reducing 
random variability (11-15). In particular, few existing prospective studies on the association between physical activity and liver cancer risk were adjusted for all relevant confounding factors, including hepatitis infection $(10,16)$. In addition, observational studies can be subject to selection bias, whereby the parameter being estimated differs from a causal effect in the total population because of differential restriction of the study population (17). Therefore, bias analysis is particularly important in meta-analyses of observational studies, where a central role is to assess the current quality of evidence. We conducted a systematic review of the literature and summarized eligible prospective studies on the association of physical activity and liver cancer risk and performed bias analyses to examine the robustness to unobserved confounding and selection bias.

\section{Methods}

\section{Literature Search}

We conducted this systematic review and meta-analysis following the Meta-analysis of Observational Studies in Epidemiology (18) guideline. Two investigators (SEB, SS) searched Medline, Embase, and Scopus from database inception to January 23, 2019, for studies that investigated the association between physical activity and the risk of liver cancer. We tailored search strategies to each database and used controlled vocabulary and search filters where available, or Boolean search methods and free-text terms. Full search terms are provided in the Supplementary Boxes 1-3 (available online). We restricted the search to human studies. No date or language restrictions were used. References of relevant publications were handsearched for additional articles.

\section{Study Selection}

Two authors (SEB, SS) independently performed the search, read the full texts of all identified articles to determine whether each study met the eligibility criteria, and resolved discrepancies by discussion. Included studies fulfilled the following criteria: 1) prospective design (ie, cohort, nested case control, case cohort, follow-up of randomized controlled trial); 2) physical activity used as an exposure variable; 3) liver cancer incidence or liver cancer mortality as outcome; 4) performance among general (healthy) populations older than 18 years without prevalent cancers at baseline; and 5) provision of hazard ratios (HRs) or relative risks (RRs) estimates with $95 \%$ confidence intervals (CIs). Studies were excluded if they measured muscle strength, physical fitness or cardiorespiratory fitness but not physical activity. If multiple studies reported on the same dataset, we included the one with the largest number of cases or longer duration of follow-up, more detailed report of physical activity, and better control of confounding variables. We considered the following to represent a minimal set of adjustment variables: age, sex, educational attainment, smoking, alcohol consumption, and coffee intake $(10,16,19)$.

\section{Data Extraction and Evaluation of Study Quality}

Data were extracted using a standardized data extraction form. Two authors (SB and SS) independently checked the data. For each of the eligible studies, the following information was extracted: first author's name, publication year, study name, population, country, age at baseline, sex, number of participants and cases, cancer site, type and intensity of physical activity, comparisons made, hazard ratios or relative risks and 95\% confidence intervals from multivariable adjusted models, and confounding factors (Table 1). Because liver cancer is relatively rare by the end of follow-up, hazard ratios were treated as estimators of relative risks (26-28). The hazard ratio and 95\% confidence interval were inverted for one study (25) that used the most active group as the reference category. A within-study inverse-variance weighted fixed-effect model was performed to obtain an overall estimate when hazard ratios were separately reported for men and women $(23,25)$.

We used the Newcastle-Ottawa Scale (29) to assess the quality of included studies consisting of representativeness of the cohort, whether the nonexposed participants were drawn from the same population as the exposed, ascertainment of the exposure, whether the outcome of interest was absent at the study onset, comparability (conditional exchangeability) of the exposed and unexposed (that is, if adjustment for the minimal adjustment set, as defined above, was made), ascertainment of the outcome, whether the length of the follow-up was long enough (at least 5 years) for the outcome to occur, and the completeness of the follow-up (loss to follow-up $<20 \%$ ). A minimum score of 2 can be awarded for comparability and a maximum of 1 can be given for each of the remaining items. A study can achieve a maximum possible quality score of 9 .

\section{Statistical Analysis}

We used the Hartung-Knapp-Sidik-Jonkman random-effects meta-analysis approach [whose advantages were demonstrated previously $(30,31)]$ to combined study-specific log hazard ratio, with the empirical Bayes estimator for the between-study variance $\left(\tau^{2}\right)(30)$, for estimation of a mean hazard ratio, with corresponding $95 \%$ confidence intervals and $95 \%$ prediction intervals (32). The $95 \%$ confidence interval from a random-effects model contains highly probable values for the mean hazard ratio $(32,33)$. The $95 \%$ prediction interval estimates where the true hazard ratio is to be expected for $95 \%$ of studies in similar settings in the future $(32,33)$. We reported the percentage of total variation due to heterogeneity $\left(\mathrm{I}^{2}\right)$; values greater than $50 \%$ to $75 \%$ are considered moderate to large (33). Cochran $Q$ statistic was used to test for between-study heterogeneity. Heterogeneity was further examined by estimating the proportion of studies in the population with evidence for a clinically meaningful effect (using thresholds for a mean $\mathrm{HR}$ of $\leq 0.7, \leq 0.8$, $\leq 0.9$ ) and the estimated proportion of studies with harmful effects (ie, HR >1) (14,32). Random-effects meta-analysis was additionally stratified by study quality [Newcastle-Ottawa Scale (29) $<6$ vs $\geq 6$ points], endpoint (incident liver cancer vs liver cancer mortality), type of physical activity (total physical activity, leisure-time physical activity, vigorous physical activity, sports participation), sex (as some studies included only male or female participants), and geographic region (Asia, Europe, United States). Because there were few studies in these strata, we did not perform meta-regression to identify sources of heterogeneity. All statistical tests were two-sided, and $P$ values less than 0.05 were considered as statistically significant.

\section{Bias Analysis}

We performed bias analysis to examine possible effects due to inclusion of small studies, selective publication of positive findings, and sensitivity to unobserved confounding and selection bias. We evaluated publication bias and small study effects 


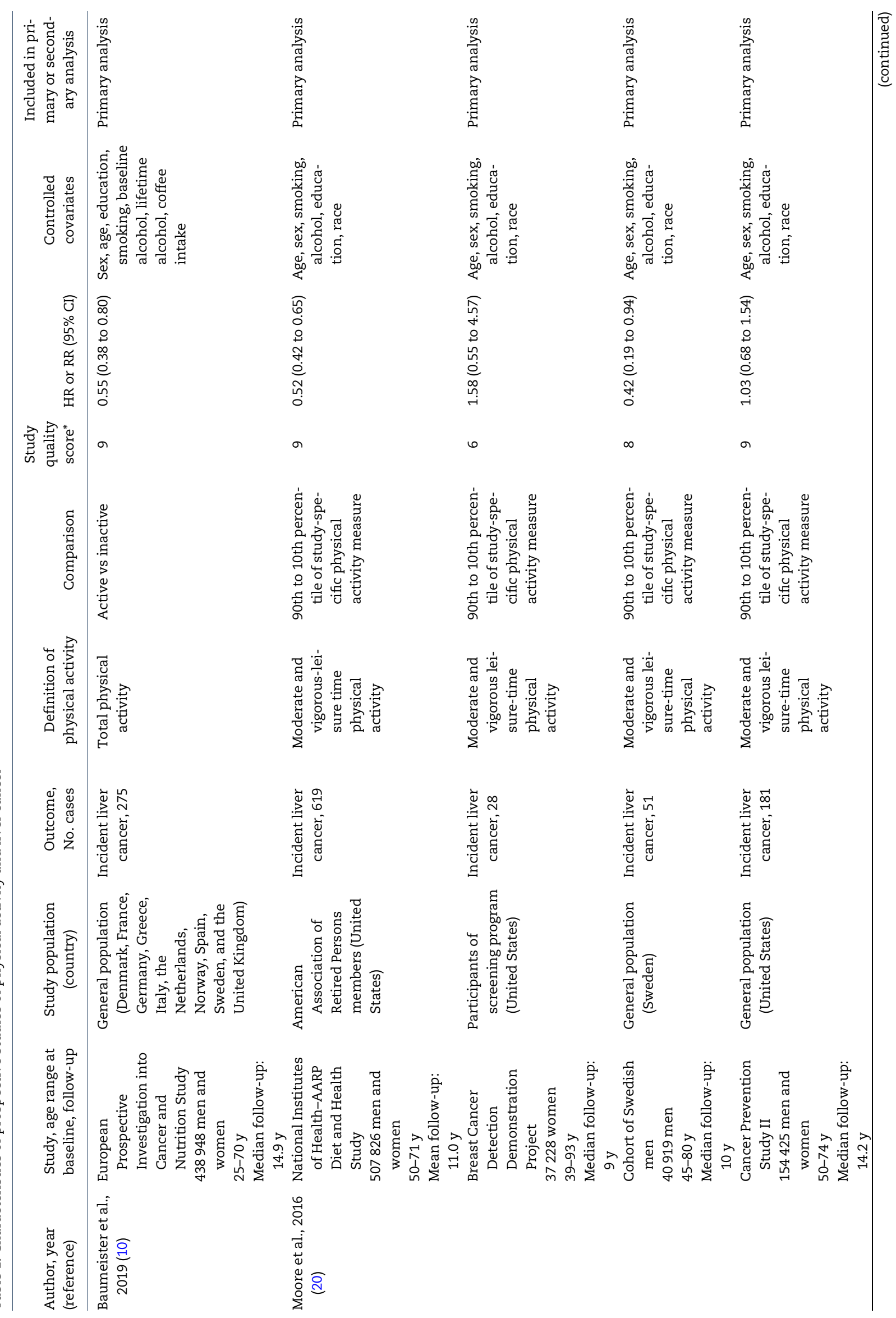




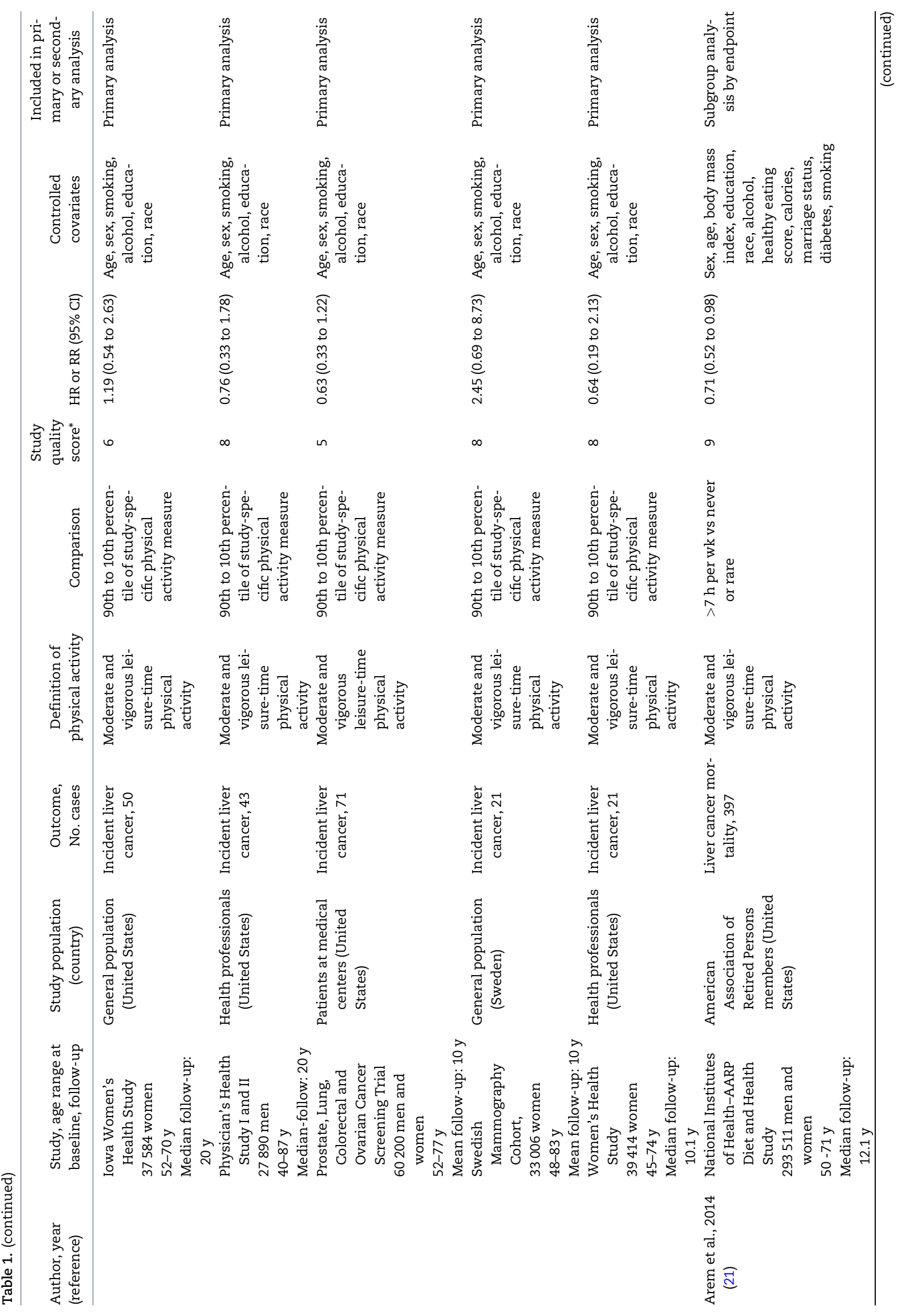




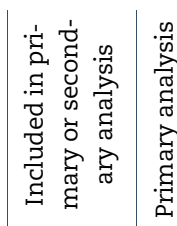

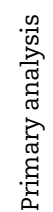

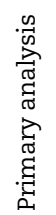

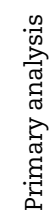

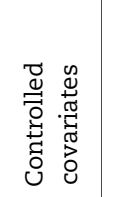

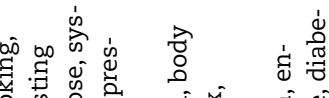

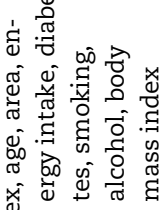

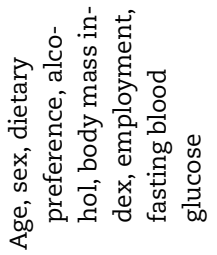

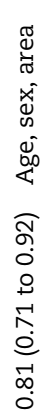

昰㤐
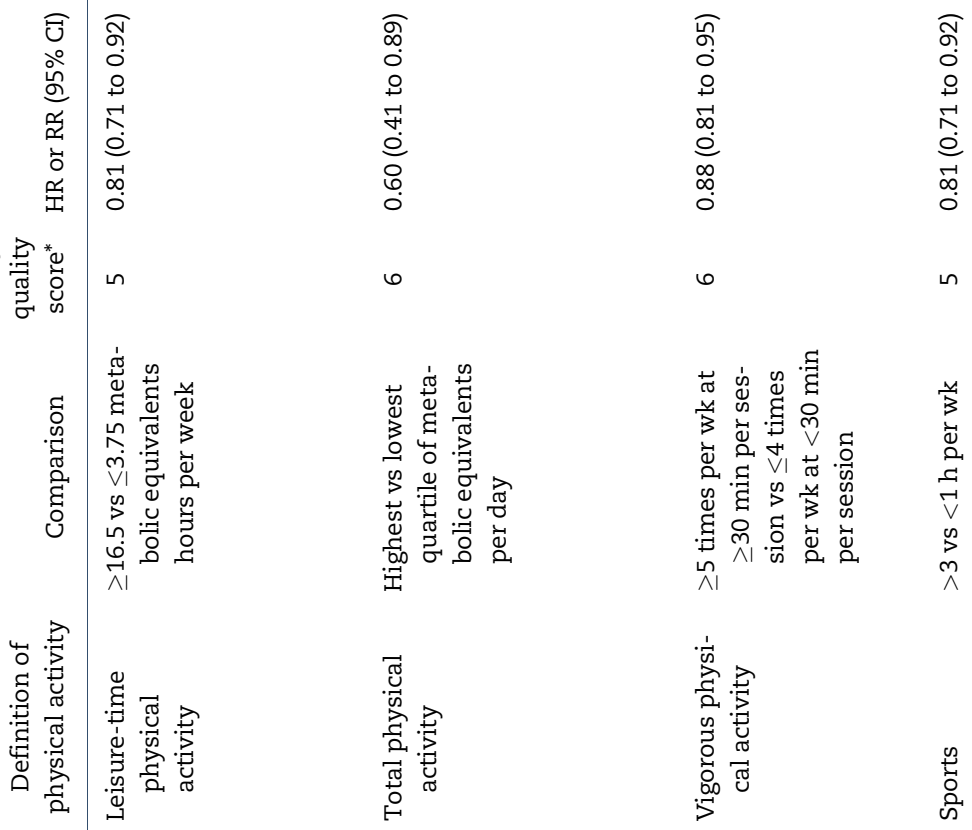

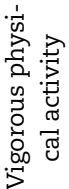

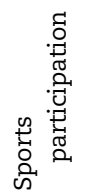
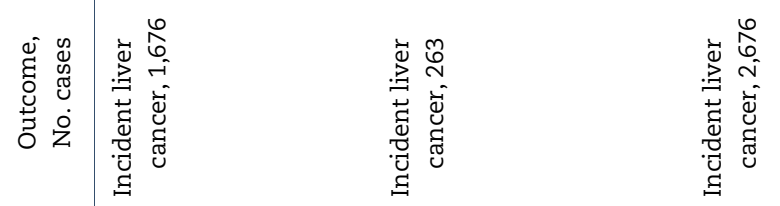

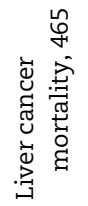
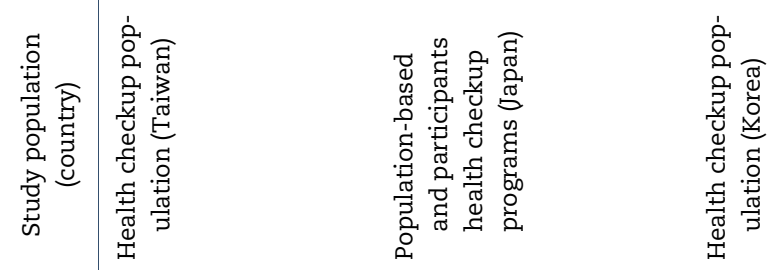

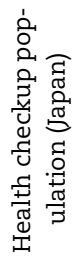

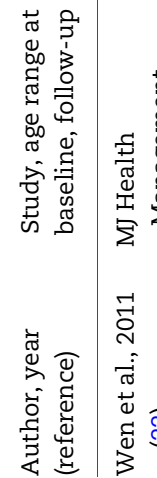
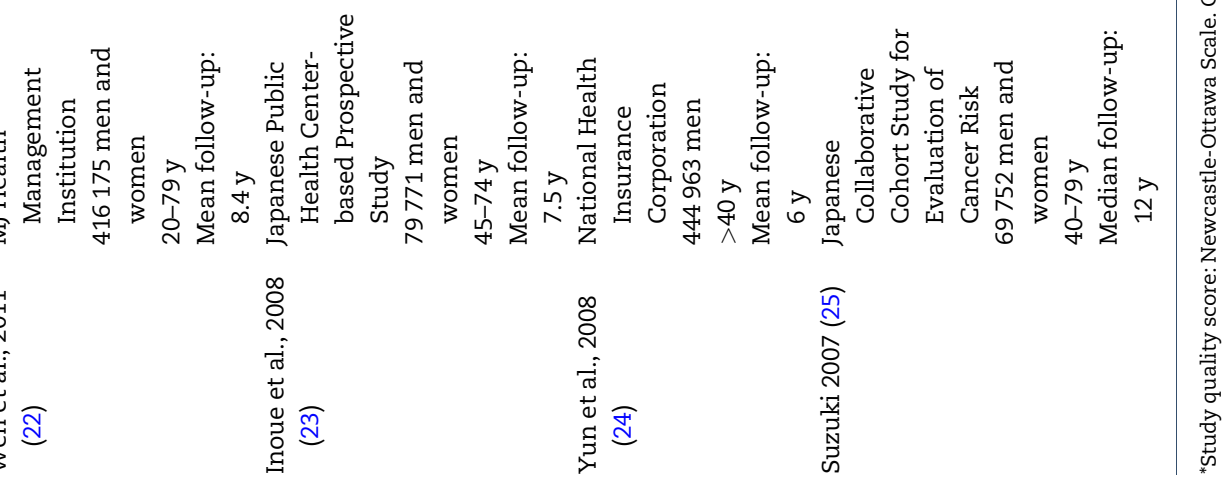
(funnel plot asymmetry) using the regression-based tests proposed by Egger and Debray (34), the trim-and-fill method (35), and the Copas selection model (35). We conducted adjustment for small study bias using the Rücker regression-based shrinkage estimator (35). Random-effects meta-analysis of observational studies can produce biased estimates of pooled effect sizes if the synthesized individual studies are subject to unmeasured confounding or selection bias (11-13). We used sensitivity analyses for unmeasured confounding in a randomeffects meta-analysis. Specially, we quantified the magnitude of unmeasured confounding capable of reducing the mean hazard ratio to the null (15). This approach to sensitivity analysis is a meta-analytic extension of the E-Value, a popular metric that quantifies the minimum strength of association that an unmeasured confounder would need to have with the exposure and the outcome on the relative risk scale to fully account for an observed exposure-outcome association, above and beyond the measured covariates (26). Given the heterogeneity of the random-effects model estimate, we additionally reported the unobserved confounding strength required to nullify $10 \%$ to $50 \%$ of studies with true hazard ratio larger than a meaningful scientific threshold (ie, HR <0.9) (15). We used an upper bounding factor approach to selection bias to determine the strength of selection on the relative risk scale that would be necessary to explain away the mean hazard ratio (17). The statistical software R [version 3.5.1, Foundation for Statistical Computing, Vienna, Austria; packages E-Value (15), meta, metafor, metamisc (34), and metasens (35)] was used.

\section{Results}

\section{Systematic Review}

Our literature search identified a total of 400 publications (Figure 1). After removal of duplicate publications, 172 studies remained for title and abstract screening, of which 22 were potentially relevant for full-text review. Among the 22 reviewed publications, studies (with possible overlap) were excluded from the primary meta-analysis on physical activity and liver cancer if the exposure variable was not physical activity $(n=6)$ $(36-41)$, or if the outcome was not liver cancer $(n=1)(19)$, or the effect estimates for physical activity were not shown $(n=4)$ $(36,37,41,42)$. Additionally, studies were excluded if the study design was not prospective $(n=5)(43-47)$ or if the article had a shorter follow-up or fewer events than another publication from the same study $(n=5)(16,41,48-50)$.

This left a total of 14 eligible studies from six publications $(10,20,22-25)$. Taken together, those studies included 2.39 million individuals and 6,440 liver cancer events or deaths. Characteristics of included studies are shown in Table 1. Self-reported physical activity, measured at baseline, served as exposure variable in all studies. The mean follow-up time was 11.6 years (range $=6$ to 20). The age of included participants ranged from 20 to 93 years (median $=45)$ at baseline examination. Among the 14 included studies, eight were conducted in the United States, two in Europe, and four in Asian countries. The quality of the studies was moderate to high for 11 studies and low for three studies.

\section{Meta-Analysis for the Association Between Physical Activity and Liver Cancer Risk}

The random-effects meta-analysis showed that physical activity is inversely associated with liver cancer risk. The mean hazard ratio for liver cancer risk, comparing high and low levels of physical activity, was 0.75 ( $95 \% \mathrm{CI}=0.63$ to 0.89 ) (Figure 2). There was moderate heterogeneity between studies (Cochran $Q$ $\left.P=.001 ; I^{2}=64.2 \%\right)$. Based on the point and $\tau^{2}$ estimates from the random-effects model, we estimated that $30.7 \%(95 \%$ $\mathrm{CI}=19.0 \%$ to $42.4 \%), 67.6 \%$ ( $95 \% \mathrm{CI}=56.6 \%$ to $78.5 \%)$, and $90.3 \%$ (95\% CI $=76.0 \%$ to $99.1 \%$ ) of all true effect estimates will have mean hazard ratios below $0.7,0.8$, and 0.9 , respectively. We further estimated that $2 \%(95 \% \mathrm{CI}=0.13 \%$ to $8.3 \%)$ of all studies have hazard ratios larger than 1.0. The $95 \%$ prediction interval for the mean hazard ratio of 0.75 was 0.52 to 1.07 ; this additionally suggests that, with high probability, a new hazard ratio drawn from the population will be less than 1 . When the metaanalysis was stratified by study quality, endpoint (incidence, mortality), type of physical activity, sex, and region, we found difference with regard to type of physical activity (Cochran $Q$ $P<.001$ ) (Table 2). Studies using total physical activity as the exposure variable revealed the largest hazard ratio, and a study that used the participation in sports as the exposure revealed the smallest hazard ratio.

\section{Bias Analysis}

There was little evidence for publication bias using analyses of funnel plot asymmetry, as indicated by Egger test $(P=.46)$ and Debray test $(P=.23)$ (Supplementary Table 1 and Figure 1, available online). We used the trim-and-fill method, Copas selection model, Rücker's shrinkage procedure, and the leave-one-out procedure to examine small study bias (Supplementary Table 2 and Figure 2, available online). The trim-and-fill method mirrored one of the cohorts included in a pooled analysis of nine US and European cohorts (20). The mean hazard ratios and $95 \%$ confidence intervals from these bias analyses were in agreement with our primary finding. Of note, when the NIH-AARP Diet and Health Study was excluded in leave-one-out analysis, heterogeneity was substantially reduced $\left(\mathrm{I}^{2}=37.2 \%, \tau^{2}=0.006\right)$, and the mean hazard ratio was $0.81(95 \% \mathrm{CI}=0.71$ to 0.92 ; prediction interval $=0.66$ to 0.98 ).

In the bias analysis for unobserved confounding, for an unmeasured confounder to explain the mean hazard ratio of 0.75 , an unobserved confounder would have to be associated with a 1.99-fold increase in the risk of physical activity or liver cancer, above and beyond the measured confounders. For an unmeasured confounder to bring the upper confidence limit of 0.89 for this mean hazard ratio above 1.0, the unmeasured confounder would have to be related to physical activity and liver cancer risk with a relative risk of 1.49 above and beyond the measured confounders. Unobserved confounder strengths with relative risk of $2.22,2.03,1.90,1.78$, and 1.68 , respectively, would be necessary to shift $10 \%, 20 \%, 30 \%, 40 \%$, and $50 \%$ of all true studies with hazard ratioless than 0.9 to 1.0 (Supplementary Figure 3, available online). By calculating the upper bounding factor $\sqrt{1 / 0.75}+\sqrt{1 / 0.75-\sqrt{1 / 0.75}}=1.58$, we come to the conclusion that unaccounted for selection variables would have to be related to physical activity and liver cancer with a relative risk of 1.58 to produce an observed hazard ratio of 0.75 if the true hazard ratio were equal to 1.0.

\section{Discussion}

The results of this meta-analysis, including 6,440 liver cancer cases from 14 prospective observational studies, indicated that high vs low physical activity reduces the risk of liver cancer by 


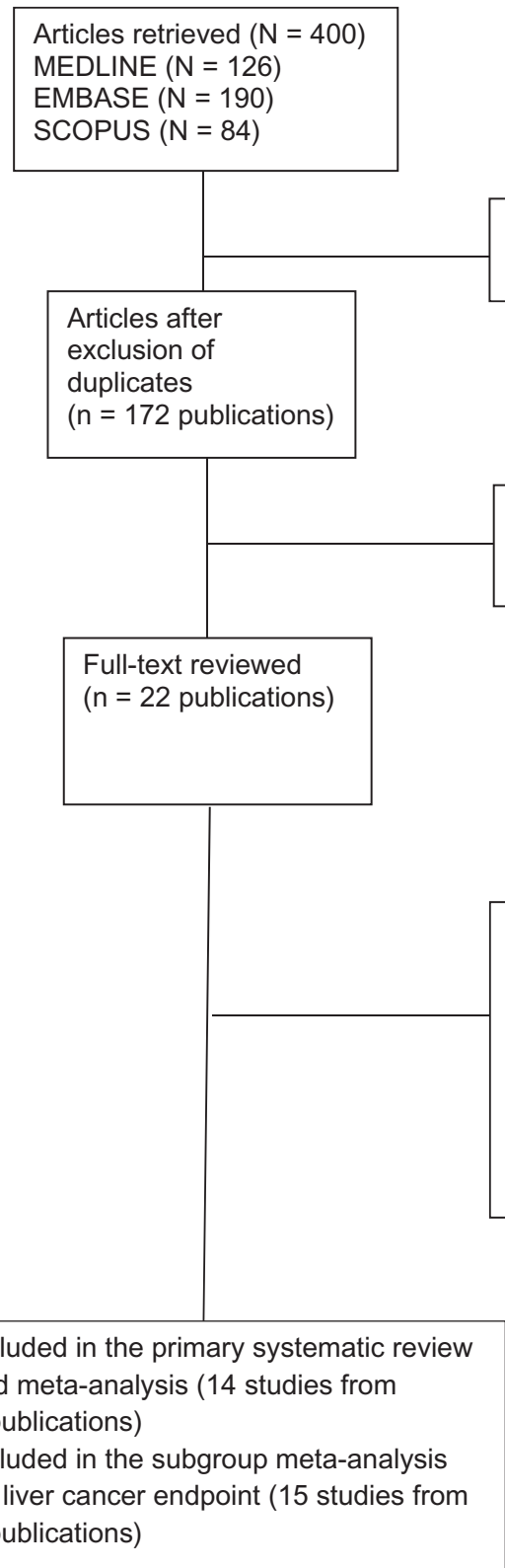

Figure 1. Flowchart of systematic review and meta-analysis selection.

$25 \%$. There was moderate heterogeneity between studies, but we estimated that $90 \%$ of all true hazard ratios would be smaller than 0.9 and $68 \%$ of all true hazard ratio would be smaller than 0.8 .

To our knowledge, one previous systematic review and meta-analysis (9) has summarized available prospective cohorts on the association of physical activity and liver cancer risk. This meta-analysis produced a mean relative risk of $0.75(95 \%$ $\mathrm{CI}=0.66$ to $0.86 ; \mathrm{I}^{2}=0 \%$ ) for the association of high vs low total physical activity and liver cancer risk, although differences in the evaluation of individual studies exist. By comparison, a pooled analysis from 10 cohorts reported a mean hazard ratio of $0.73\left(95 \% \mathrm{CI}=0.55\right.$ to $\left.0.98 ; I^{2}=55 \%\right)(20)$. Of note, the metaanalysis (9) and the pooled analysis (20) did not include the most recent pan-European cohort study on physical activity and liver cancer risk (10).
Excluded duplicates $(\mathrm{n}=185)$

Excluded based on title and

abstract

$(n=150)$

\section{Excluded based on full text}

Reasons (with possible overlap):

Exposure was not physical activity $(n=6)$

Outcome was not liver cancer $(n=1)$

Effect estimates for physical activity not

shown $(n=4)$

Study design not prospective cohort $(n=5)$

Publication with longer follow-up or more

events from the same study available $(n=5)$
A concern of our meta-analysis includes the potential for uncontrolled confounding. Although most estimates in the individual studies were adjusted for relevant confounders, metaanalysis of observational associations can be biased because of residual confounding. Most notably, chronic infections with hepatitis virus $B$ and $C$ are strong liver cancer risk factors and account for more than $70 \%$ of liver cancers worldwide $(3,4)$. Very few data from prospective settings are available from populations with high hepatocellular carcinoma incidence and a greater contribution of chronic hepatitis infection (10). Accordingly, because of the potential for residual confounding by unmeasured confounders in several of the individual studies, we performed sensitivity analysis to quantify the extent to which associations across studies were robust to unmeasured confounding. We found that a modestly strong unobserved confounder, with a 1.99-fold increase of the risk of the exposure or 
Study

Baumeister, et al., 2019 (10)

Moore, et al.,2016 (20)

Moore, et al.,2016 (20)

Moore, et al.,2016 (20)

Moore, et al.,2016 (20)

Moore, et al.,2016 (20)

Moore, et al.,2016 (20)

Moore, et al.,2016 (20)

Moore, et al.,2016 (20)

Moore, et al.,2016 (20)

Wen, et al., 2011 (22)

Inou, et al., 2008 (23)

Yun, et al., 2008 (24)

Suzuki, et al., 2007 (25)
Cohort Events

$\begin{array}{rr}\text { EPIC } & 275 \\ \text { AARP } & 619 \\ \text { CPSII } & 181 \\ \text { PLCO } & 71 \\ \text { COSM } & 51 \\ \text { IWHS } & 50 \\ \text { PHS } & 43 \\ \text { BCDDP } & 28 \\ \text { SMC } & 21 \\ \text { WHS } & 21 \\ \text { MJHMI } & 1676 \\ \text { JPHCPS } & 263 \\ \text { NHIC } & 2676 \\ \text { JCCSECR } & 465\end{array}$

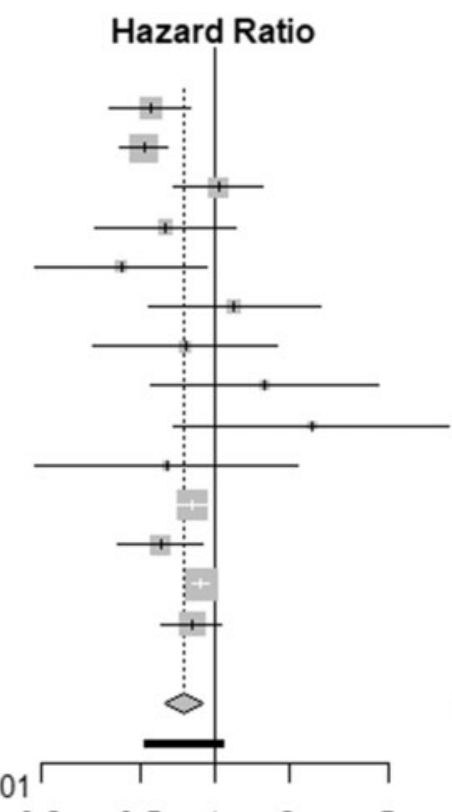

$\begin{array}{lllll}0.2 & 0.5 & 1 & 2 & 5\end{array}$
HR $\quad 95 \%-\mathrm{Cl}$ Weight

$0.55 \quad[0.38 ; 0.80] \quad 8.0 \%$

$0.52 \quad[0.42 ; 0.65] \quad 13.7 \%$

$1.03 \quad[0.68 ; 1.55] \quad 7.1 \%$

$0.63 \quad[0.33 ; 1.21] \quad 3.4 \%$

$0.42 \quad[0.19 ; 0.93] \quad 2.4 \%$

$1.19 \quad[0.54 ; 2.63] \quad 2.5 \%$

$0.76 \quad[0.33 ; 1.77] \quad 2.2 \%$

$1.58 \quad[0.55 ; 4.55] \quad 1.5 \%$

$2.45 \quad[0.69 ; 8.71] \quad 1.0 \%$

$0.64 \quad[0.19 ; 2.14] \quad 1.1 \%$

$0.81 \quad[0.71 ; 0.92] \quad 18.1 \%$

$0.60 \quad[0.41 ; 0.89] \quad 7.5 \%$

$0.88 \quad[0.81 ; 0.95] \quad 20.3 \%$

$0.81 \quad[0.61 ; 1.07] \quad 11.1 \%$

\section{$0.75[0.63 ; 0.89] 100.0 \%$}

$[0.52 ; 1.07]$

\section{High vs. low physical activity}

Figure 2. Forest plot from random-effects meta-analysis of prospective studies on the risk of liver cancer comparing high and low levels of physical activity. Liver cancer was defined as HCC (hepatocellular carcinoma, C22.0) and/or IHBC (intrahepatic bile duct cancer, C22.1). Study-specific hazard ratios (HR) are represented by black diamonds (with their 95\% confidence interval [CI] as error bars). hazard ratios were combined using a Hartung-Knapp-Sidik-Jonkman random-effects model, yielding a mean hazard ratio and its $95 \%$ confidence interval and $95 \%$ prediction interval. The dotted line represents the mean hazard ratio. Two-sided $P$ value for between-study heterogeneity based on Cochran Q statistic. AARP $=$ National Institutes of Health-AARP Diet and Health Study; BCDDP $=$ Breast Cancer Detection Demonstration Project; COSM = Cohort of Swedish Men; CPSII = Cancer Prevention Study II; EPIC = European Prospective Investigation into Cancer and Nutrition Study; IWHS = Iowa Women's Health Study; JCCSECR = Japanese Collaborative Cohort Study for Evaluation of Cancer Risk; JPHCPS = Japanese Public Health Center-based Prospective Study; MJHMI = MJ Health Management Institution; NHIC = National Health Insurance Corporation; PHS = Physician's Health Study I and II; PLCO = Prostate, Lung, Colorectal and Ovarian Cancer Screening Trial; SMC = Swedish Mammography Cohort; WHS = Women's Health Study.

Table 2. Subgroup meta-analyses of physical activity and liver cancer*

\begin{tabular}{|c|c|c|c|c|c|}
\hline Subgroup & No. of studies & $\mathrm{HR}(95 \% \mathrm{CI}) \dagger$ & $I^{2}, \%$ & $\tau^{2}$ & $\mathrm{P}_{\mathrm{h} 1} \neq$ \\
\hline Study quality & & & & & .42 \\
\hline NOS $\geq 6$ & 11 & 0.73 (0.57 to 0.94$)$ & 72.0 & 0.032 & \\
\hline NOS $<6$ & 3 & 0.80 (0.70 to 0.92$)$ & 0 & 0 & \\
\hline Endpoint & & & & & 0.45 \\
\hline Incident liver cancer & 13 & 0.74 (0.61 to 0.90$)$ & 67.0 & 0.023 & \\
\hline Liver cancer mortality & 3 & $0.80(0.68 ; 0.94)$ & 0 & 0 & \\
\hline \multicolumn{6}{|l|}{ Type of physical activity } \\
\hline Total physical activity & 2 & 0.57 (0.44 to 0.75$)$ & 0 & 0 & $<.001$ \\
\hline Leisure-time physical activity & 10 & 0.75 (0.67 to 0.83$)$ & 61.5 & 0.0364 & \\
\hline Vigorous physical activity & 1 & $0.88(0.81,0.95)$ & 0 & 0 & \\
\hline Sports participation & 1 & 0.81 (0.61 to 1.07$)$ & 0 & 0 & \\
\hline Sex & & & & & .26 \\
\hline Men§ & 5 & 0.86 (0.74 to 1.00$)$ & 29.0 & $<0.001$ & \\
\hline Women $\|$ & 6 & 0.87 (0.50 to 1.52$)$ & 28.6 & 0.031 & \\
\hline Men and womenף & 5 & 0.68 (0.48 to 0.97$)$ & 76.2 & 0.032 & \\
\hline Geographic region & & & & & .56 \\
\hline Asia & 4 & 0.85 (0.80 to 0.91$)$ & 31.1 & $<0.001$ & \\
\hline Europe & 3 & 0.62 (0.43 to 0.88$)$ & 79.6 & 0.096 & \\
\hline United States & 7 & 0.64 (0.54 to 0.75$)$ & 53.5 & 0.052 & \\
\hline
\end{tabular}

"Liver cancer was defined as HCC (hepatocellular carcinoma, C22.0) and/or IHBC (intrahepatic bile duct cancer, C22.1). CI = confidence interval; HR = hazard ratio; NOS $=$ Newcastle-Ottawa Scale; $I^{2}=$ percentage of total variance explained by $\tau^{2} ; \tau^{2}=$ between-study variance.

†Hazard ratios were combined using a Hartung-Knapp-Sidik-Jonkman random-effects model.

$\ddagger$ Two-sided $P$ value was calculated using $Q$ test for subgroup differences.

§Primary study included only men or reported HR for men.

$\|$ Primary study included only women or reported HR for women.

१Primary study included men and women and reported one HR for men and women. 
outcome, would suffice to explain away the mean hazard ratio of 0.75 . A confounder strength of a 1.49-fold increased risk of exposure and outcome would suffice to render the estimate statistically nonsignificant. Individuals with hepatitis B virus and hepatitis $C$ virus infections show a 15 - to 20 -fold increase in the risk of hepatocellular carcinoma compared with seronegative individuals (51), indicating the potential for unobserved confounding by hepatitis virus infection to account for the observed association between physical activity and liver cancer risk. Selection bias with a 1.58-fold increased risk for unaccounted for selection variables would be sufficient to shift the observed hazard ratio to the null. Thus, a potential role for chronic hepatitis infection and other unobserved confounders and selection factors (such as lack of access to medical care or adverse socioeconomic conditions associated with cohort participation) remains to bias the observed association of physical activity and liver cancer risk.

Limitations of our meta-analysis include that individual studies relied on self-reported measures of physical activity, which may introduce measurement error and potentially biased effect estimates. Self-reported measures of activity may be affected by mood states, social desirability, or cognitive biases that also may affect liver cancer liability. However, a majority of the studies used physical activity questionnaires that underwent rigorous validation against objective measures of energy expenditure and cardiorespiratory fitness. A further concern is that assessments of type, duration, and intensity of physical activity differed somewhat by study. In addition, not all studies assessed moderate and vigorous physical activities separately. Because the majority of the included studies reported risk estimates for high vs low levels of physical activity, we could not examine intensities of physical activity required to reduce liver cancer risk using dose-response meta-analysis. Finally, only one included study (10) reported effect estimates on types of hepatobiliary cancers, which precluded analyses by cancer subtype.

A large number of experimental and observational studies suggest that the most important mechanism by which physical activity affects gastrointestinal cancer occurrence is lowering body weight (visceral fatness in particular) $(52,53)$. The exact biologic mechanisms underlying the association between adiposity and liver cancer are not fully established, but main mechanisms include dysregulation of adipose tissue-derived inflammation (immune-related and other), alterations in insulin signaling, growth hormone pathways, and sex hormone metabolism (53-55).

In conclusion, this quantitative synthesis of all available prospective studies suggests that physical activity reduces the risk of liver cancer. This study leveraged quantitative bias analysis to test how much unobserved confounding and selection bias would suffice to bias effect estimates. Moderately strong unobserved confounding and selection bias suffice to explain away the observed inverse association between physical activity and liver cancer risk. Studies with more detailed and objectively measured physical activity assessed at multiple time points throughout the life course, and adjustment for hepatitis infection, are needed to provide additional evidence on this association. Recent developments in quasi-experimental designs $(56,57)$ offer the opportunity to further reduce potential confounding bias. Stronger evidence for causal associations is of great importance because few modifiable factors for preventing liver cancer are known.

\section{Funding}

This research did not receive any grant from funding agencies in the public, commercial, or not-for-profit sectors.

\section{Notes}

Affiliations of authors: Epidemiology, LMU München, UNIKA-T Augsburg, Augsburg, Germany (SEB, JL); Independent Research Group Clinical Epidemiology, Helmholtz Zentrum München, German Research Center for Environmental Health, Munich, Germany (SEB, JL); Institute for Community Medicine, University Medicine Greifswald, Greifswald, Germany (SEB); Department of Epidemiology and Preventive Medicine, University of Regensburg, Germany (MFL); Junior Research Group Systematic Reviews, Institute for Biometrics and Epidemiology, German Diabetes Center, Leibniz Center for Diabetes Research at Heinrich Heine University, Düsseldorf, Germany (SS)

All authors disclose no conflict. We thank all study participants and members of the study teams of the included studies.

\section{References}

1. Akinyemiju T, Abera S, Ahmed M, et al. The burden of primary liver cancer and underlying etiologies from 1990 to 2015 at the global, regional, and national level: results from the Global Burden of Disease Study 2015. JAMA Oncol. 2017;3(12):1683-1691.

2. Choo SP, Tan WL, Goh BKP, et al. Comparison of hepatocellular carcinoma in Eastern versus Western populations. Cancer. 2016;122(22):3430-3446.

3. Llovet JM, Zucman-Rossi J, Pikarsky E, et al. Hepatocellular carcinoma. Nat Rev Dis Primers. 2016;2:16018.

4. Saran U, Humar B, Kolly P, et al. Hepatocellular carcinoma and lifestyles. J Hepatol. 2016;64(1):203-214.

5. Ozakyol A. Global epidemiology of hepatocellular carcinoma (HCC epidemiology). J Gastrointest Cancer. 2017;48(3):238-240.

6. Rezende LFM, Sa TH, Markozannes G, et al. Physical activity and cancer: an umbrella review of the literature including 22 major anatomical sites and 770000 cancer cases. Br J Sports Med. 2018;52(13):826-833.

7. World Cancer Research Fund International, American Institute for Cancer Research. Diet, Nutrition, Physical Activity and Cancer: A Global Perspective. Third Expert Report. London, UK: World Cancer Research Fund International; 2018.

8. Giovannucci E. Nutritional epidemiology and cancer: a tale of two cities. Cancer Causes Control. 2018;29(11):1007-1014.

9. Lin ZZ, Xu YC, Liu CX, et al. Physical activity and liver cancer risk: a systematic review and meta-analyses [published online ahead of print October 26 2018]. Clin J Sport Med 2018. doi:10.1097/jsm.0000000000000689.

10. Baumeister SE, Schlesinger S, Aleksandrova K, et al. Association of physical activity and risk of hepatobiliary cancers: a multinational cohort study. J Hepatol. 2019;70(5):885-892.

11. Shrier I, Boivin JF, Steele RJ, et al. Should meta-analyses of interventions include observational studies in addition to randomized controlled trials? A critical examination of underlying principles. Am J Epidemiol. 2007;166(10): 1203-1209.

12. Egger M, Schneider M, Davey Smith G. Spurious precision? Meta-analysis of observational studies. BMJ. 1998;316(7125):140-144.

13. Valentine JC, Thompson SG. Issues relating to confounding and metaanalysis when including non-randomized studies in systematic reviews on the effects of interventions. Res Synth Methods. 2013;4(1):26-35.

14. Mathur MB, VanderWeele TJ. New metrics for meta-analyses of heterogeneous effects. Stat Med. 2019;38(8):1336-1342.

15. Mathur MB, VanderWeele TJ. Sensitivity analysis for unmeasured confounding in meta-analyses [published online ahead of print April 30, 2019]. J Am Stat Assoc. doi.org/10.1080/01621459.2018.1529598.

16. Behrens G, Matthews CE, Moore SC, et al. The association between frequency of vigorous physical activity and hepatobiliary cancers in the NIH-AARP Diet and Health Study. Eur J Epidemiol. 2013;28(1):55-66.

17. Smith LH, VanderWeele TJ. Bounding bias due to selection. Epidemiology. 2019;30(4):509-516.

18. Stroup DF, Berlin JA, Morton SC, et al. Meta-analysis of observational studies in epidemiology: a proposal for reporting. Meta-analysis Of Observational Studies in Epidemiology (MOOSE) group. JAMA. 2000;283(15):2008-2012.

19. Keum N, Bao Y, Smith-Warner SA, et al. Association of physical activity by type and intensity with digestive system cancer risk. JAMA Oncol. 2016;2(9): 1146-1153. 
20. Moore SC, Lee IM, Weiderpass E, et al. Association of leisure-time physical activity with risk of 26 types of cancer in 1.44 million adults. JAMA Intern Med. 2016;176(6):816-825.

21. Arem H, Moore SC, Park Y, et al. Physical activity and cancer-specific mortality in the NIH-AARP Diet and Health Study cohort. Int J Cancer. 2014;135(2): 423-431.

22. Wen CP, Wai JP, Tsai MK, et al. Minimum amount of physical activity for reduced mortality and extended life expectancy: a prospective cohort study. Lancet. 2011;378(9798):1244-1253.

23. Inoue $\mathrm{M}$, Yamamoto $\mathrm{S}$, Kurahashi $\mathrm{N}$, et al. Daily total physical activity level and total cancer risk in men and women: results from a large-scale population-based cohort study in Japan. Am J Epidemiol. 2008;168(4):391-403.

24. Yun YH, Lim MK, Won YJ, et al. Dietary preference, physical activity, and cancer risk in men: national health insurance corporation study. BMC Cancer. 2008;8(366):

25. Suzuki K. Health conditions and mortality in the Japan Collaborative Cohor Study for Evaluation of Cancer (JACC). Asian Pac J Cancer Prev. 2007;8(suppl): $25-34$

26. VanderWeele TJ, Ding P. Sensitivity analysis in observational research: introducing the E-value. Ann Intern Med. 2017;167(4):268-274.

27. Tierney JF, Stewart LA, Ghersi D, et al. Practical methods for incorporating summary time-to-event data into meta-analysis. Trials. 2007;8:16.

28. Higgins JPT, Green S, eds. Cochrane Handbook for Systematic Reviews of Interventions Version 5.1.0 [updated March 2011]. The Cochrane Collaboration, 2011. Available from http://handbook.cochrane.org.

29. Wells G, Shea B, O'Connell D, et al. The Newcastle-Ottawa Scale (NOS) for assessing the quality of nonrandomised studies in meta-analyses. 2011. www.ohri.ca/programs/clinical_epidemiology/. Accessed December 31, 2018.

30. Veroniki AA, Jackson D, Bender R, et al. Methods to calculate uncertainty in the estimated overall effect size from a random-effects meta-analysis. Res Synth Methods. 2019;10(1):23-43.

31. IntHout J, Ioannidis JP, Borm GF. The Hartung-Knapp-Sidik-Jonkman method for random effects meta-analysis is straightforward and considerably outperforms the standard DerSimonian-Laird method. BMC Med Res Methodol. 2014;14:25.

32. IntHout J, Ioannidis JP, Rovers MM, et al. Plea for routinely presenting prediction intervals in meta-analysis. BMJ Open. 2016;6(7):e010247.

33. Higgins JP, Thompson SG, Spiegelhalter DJ. A re-evaluation of randomeffects meta-analysis. J R Stat Soc Ser A Stat Soc. 2009;172(1):137-159.

34. Debray TPA, Moons KGM, Riley RD. Detecting small-study effects and funnel plot asymmetry in meta-analysis of survival data: a comparison of new and existing tests. Res Synth Methods. 2018;9(1):41-50

35. Schwarzer G, Carpenter JR, Rücker G. Small-study effects in meta-analysis In: Schwarzer G, Carpenter JR, Rücker G, eds. Meta-Analysis with R. Cham Heidelberg New York Dordrecht London: Springer; 2015:107-141.

36. Batty GD, Shipley MJ, Jarrett RJ, et al. Obesity and overweight in relation to organ-specific cancer mortality in London (UK): findings from the original Whitehall study. Int J Obes. 2005;29(10):1267-1274.

37. Leung WW, Ho SC, Chan HL, et al. Moderate coffee consumption reduces the risk of hepatocellular carcinoma in hepatitis B chronic carriers: a casecontrol study. J Epidemiol Community Health. 2011;65(6):556-558.

38. Peel JB, Sui X, Matthews CE, et al. Cardiorespiratory fitness and digestive cancer mortality: findings from the aerobics center longitudinal study. Cancer Epidemiol Biomarkers Prev. 2009;18(4):1111-1117.
39. Rusiecki J, Thomas D, Blair A. Mortality among United States Coast Guard marine inspectors: a follow up. Mil Med. 2009;174(8):843-851.

40. Thompson AM, Church TS, Janssen I, et al. Cardiorespiratory fitness as a predictor of cancer mortality among men with pre-diabetes and diabetes. Diabetes Care. 2008;31(4):764-769.

41. Wen CP, Lin J, Yang YC, et al. Hepatocellular carcinoma risk prediction model for the general population: the predictive power of transaminases. $J$ Nat Cancer Inst. 2012;104(20):1599-1611.

42. Romaguera D, Vergnaud AC, Peeters PH, et al. Is concordance with World Cancer Research Fund/American Institute for Cancer Research guidelines for cancer prevention related to subsequent risk of cancer? Results from the EPIC study. Am J Clin Nutr. 2012;96(1):150-163.

43. Ahmadi B, Alimohammadian M, Yaseri M, et al. Multimorbidity: epidemiology and risk factors in the Golestan Cohort Study, Iran: a cross-sectional analysis. Medicine (Baltimore). 2016;95(7):e2756.

44. Bastani R, Glenn BA, Maxwell AE, et al. Cluster-randomized trial to increase hepatitis B testing among Koreans in Los Angeles. Cancer Epidemiol Biomarkers Prev. 2015;24(9):1341-1349.

45. Konishi M, Kondou H, Okada K. Health status, life habits, and social background among the JPHC study participants at baseline survey. Japan Public Health Center-based Prospective Study on Cancer and Cardiovascular Diseases. J Epidemiol. 2001;11(6 suppl):57-74.

46. Pukkala E, Martinsen JI, Lynge E, et al. Occupation and cancer-follow-up of 15 million people in five Nordic countries. Acta Oncol. 2009;48(5):646-790.

47. Velasco-Contreras ME. Health profile of Instituto Mexicano del Seguro Social workers [in Spanish]. Rev Med Inst Mex Seguro Soc. 2013;51(1):12-25.

48. Arem H, Loftfield E, Saint-Maurice PF, et al. Physical activity across the lifespan and liver cancer incidence in the NIH-AARP Diet and Health Study cohort. Cancer Med. 2018;7(4):1450-1457.

49. Lo FE, Lu PJ, Tsai MK, et al. The role of physical activity in harm reduction among betel quid chewers from a prospective cohort of 419,378 individuals. PLoS One. 2016;11(4):e0152246.

50. Ukawa S, Tamakoshi A, Wakai K, et al. Associations of daily walking and television viewing time with liver cancer mortality: findings from the Japan Collaborative Cohort Study. Cancer Causes Control. 2014;25(7):787-793.

51. El-Serag HB. Epidemiology of viral hepatitis and hepatocellular carcinoma. Gastroenterology. 2012;142(6):1264-1273.e1.

52. Giovannucci E. An integrative approach for deciphering the causal associations of physical activity and cancer risk: the role of adiposity. J Natl Cancer Inst. 2018;110(9):935-941.

53. Giovannucci E. A framework to understand diet, physical activity, body weight, and cancer risk. Cancer Causes Control. 2018;29(1):1-6.

54. Murphy N, Jenab M, Gunter MJ. Adiposity and gastrointestinal cancers: epidemiology, mechanisms and future directions. Nat Rev Gastroenterol Hepatol. 2018;15(11):659-670.

55. Ruiz-Casado A, Martin-Ruiz A, Perez LM, et al. Exercise and the hallmarks of cancer. Trends Cancer. 2017;3(6):423-441.

56. Hamer M, Bauman A, Bell JA, et al. Examining associations between physical activity and cardiovascular mortality using negative control outcomes [published online ahead of print December 11, 2018]. Int J Epidemiol. 2018. doi: 10.1093/ije/dyy272.

57. Choi KW, Chen CY, Stein MB, et al. Assessment of bidirectional relationships between physical activity and depression among adults: a 2-sample Mendelian randomization study. JAMA Psychiatry. 2019;76(4):399-408. 